Running Head: PERSONAL GROWTH AND LIFE SATISFACTION ACROSS TIME

\title{
Is Personal Growth Initiative Associated with Later Life Satisfaction in Chinese College Students?: A 15-Week Prospective Analysis
}

\author{
Edward C. Chang ${ }^{1}$, Hongfei Yang ${ }^{2}$, Shangwen $\mathrm{Yi}^{3}$, Fei Xie ${ }^{3}$, Jiting Liu ${ }^{3}$, Haining Ren ${ }^{3}$, Jiaqi \\ Zhang ${ }^{3}$, Zhuoran Zhang ${ }^{3}$, Runzhe $\mathrm{Wu}^{3}$, Yijing Lin ${ }^{3}$, Mingqi $\mathrm{Li}^{4}$, Kaidi $\mathrm{Wu}^{1}, \mathrm{Ka} \mathrm{Ip}^{1}$, Abigael G. \\ Lucas ${ }^{1}, \&$ Olivia D. Chang ${ }^{1}$ \\ ${ }^{1}$ University of Michigan, USA, ${ }^{2}$ Zhejiang University, China, ${ }^{3}$ Beijing Normal University, China, \\ ${ }^{4}$ DePaul University, USA

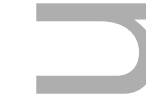 \\ Do Not Cite Without Permission from First Author. \\ Revision submitted to Asian Journal of Social Psychology. (3/24/19). \\ WORD COUNT: 4278 words
}

Q

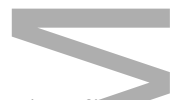

AUTHOR'S NOTE

The first author would like to acknowledge Tae Myung-Sook and Chang Suk-Choon for their encouragement and support throughout this project. The third through eleventh co-authors contributed equally to the project. Their authorship order was determined randomly.

Correspondence concerning this article should be sent to Edward C. Chang or Olivia D. Chang, Department of Psychology, University of Michigan, 530 Church Street, Ann Arbor, MI 48109. Electronic mail may be sent to changec@umich.edu or ochang@umich.edu.

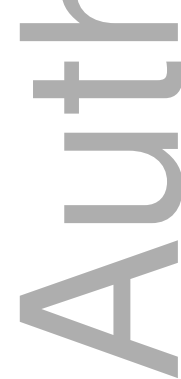

This is the author manuscript accepted for publication and has undergone full peer review but has not been through the copyediting, typesetting, pagination and proofreading process, which may lead to differences between this version and the Version of Record. Please cite this article as doi: $\underline{10.1111 / A J S P .12386}$

This article is protected by copyright. All rights reserved 
DR. EDWARD CHIN-HO CHANG (Orcid ID : 0000-0002-9179-8542)

Article type : Short Note

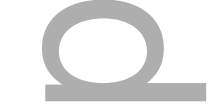

-

\section{Abstract}

Personal growth initiative is presumed to foster positive change leading to positive psychological adjustment. Accordingly, in this study we examined personal growth initiative as a predictor of life satisfaction 15 weeks later in a sample of 152 Chinese college students. Time 1 personal growth initiative was found to explain a significant amount of unique variance in Time 2 life satisfaction, even after controlling for Time 1 life satisfaction and Time 2 personal growth initiative. Specifically, (lower) intentional behavior and planfulness at baseline emerged as significant predictors of later life satisfaction. No evidence was found indicating that life satisfaction at Time 1 accounted for any significant amount of unique variance in personal growth initiative processes at Time 2, after controlling for baseline personal growth initiative processes and concurrent life satisfaction. Overall, the present findings are the first to demonstrate the importance of personal growth initiative as a prospective predictor of positive psychological adjustment in adults.

Keywords: Chinese; Personal Growth Initiative; Life Satisfaction; College Students: Prospective Design

\section{Is Personal Growth Initiative Associated with Later Life Satisfaction in Chinese College Students?: A 15-Week Prospective Analysis}

With the growth of positive psychology over the past two decades (e.g., Chang, Downey, Hirsch, \& Lin, 2016; Seligman \& Csikszentmihalyi, 2000), it is not surprising that researchers have focused on trying to identify positive psychological processes that might be linked to psychological adjustment. Indeed, consistent with the idea that personal growth plays a key role in promoting mental health, findings from studies have shown that personal growth initiative in adults is correlated with adjustment (e.g., positive affect, psychological well-being; Robitschek 
\& Keyes, 2009; Shorey, Little, Snyder, Kluck, \& Robitschek, 2007; Stevic \& Ward, 2008).

According to Robitschek (1998), personal growth initiative is viewed as an active and purposeful skill-based process that helps individuals achieve positive change and development, and is measured by the Personal Growth Initiative Scale (PGIS). Recently, however, the Personal Growth Initiative Scale-II (PGIS-II) was developed by Robitschek et al. (2012) to better account for the distinct facets of personal growth initiative. Based on their updated model, personal growth initiative is believed to be made up of four dimensions, namely, readiness for change, planfulness, using resources, and intentional behavior. Readiness for change involves one's preparedness for making specific changes in oneself. Planfulness involves one's ability to make effective plans to facilitate growth. Using resources involves one's ability to capitalize on available resources to facilitate positive personal growth. Finally, intentional behavior involves the conscious and constant pursuit of personal growth. According to Robitschek et al. (2012), readiness and planfulness are believed to tap into cognitive aspects of personal growth initiative, whereas using resources and intentional behavior are believed to tap into behavioral aspects of personal growth initiative.

Given personal growth initiative (PGI) theory, findings from recent studies using the PGIS-II in adults from different cultural backgrounds (e.g., US, China) have shown that it is indeed tapping into a multifaceted construct (e.g., Chang \& Yang, 2016; Robitschek et al., 2012). For example, Yang and Chang (2014) were able to replicate the four-dimensional structure of the PGIS-II in a sample of Chinese students. Moreover, findings from studies have also identified a reliable positive association between personal growth initiative and markers of positive psychological adjustment such as life satisfaction (Diener, 1984). For example, Yang and Chang (2014) also found that personal growth initiative was associated with greater life satisfaction in adults from both the US and China. Yet, a major limitation across current studies indicating the presence of a positive association between personal growth initiative and positive adjustment has been a reliance on using a cross-sectional design. That is, it is unclear if personal growth initiative might be prospectively associated with changes in life satisfaction, life satisfaction might be prospectively associated with changes in personal growth initiative, or both. Accordingly, the present study was conducted to clarify the association between personal growth initiative and life satisfaction across time. In turn, understanding how these variables relate to each other across time help determine the potential usefulness of helping adults engage 
in greater personal growth initiative processes, experience greater life satisfaction, or both. Purpose of the Present Study

The major objectives of the present study were to (a) examine if personal growth initiative in college students predicts changes in life satisfaction across time; and (b) examine if life satisfaction in college students predicts changes in personal growth initiative across time.

Consistent with the notion that personal growth initiative represents a positive skill-based psychological construct that facilitates positive self-change and development (Robitschek, 1998; Robitschek et al., 2012), we hypothesized that personal growth initiative in students would predict significant variance in subsequent life satisfaction, even after controlling for baseline life satisfaction and subsequent personal growth initiative. Given the reliable positive association previously found between planfulness and life satisfaction in diverse student groups (e.g., American students, Chinese students; Yang \& Chang, 2014, 2016), we hypothesized that within the personal growth initiative set, planfulness would represent a unique prospective predictor of subsequent life satisfaction in students.

In contrast, we hypothesized that life satisfaction would not predict changes in personal growth initiative across time. That is, for individuals who are satisfied with their life, there may be no compelling motivation for them to seek purposeful change in their future. Indeed, consistent with this view, Luhmann and Hennecke (2017) found that individuals who were asked to identify things that made their life positive were not more motivated to seek change compared to a control group. Interestingly, however, these researchers found that individuals asked to identify things that made their life negative were more motivated to seek change compared to a control group.

\section{Method}

\section{Participants}

Participants were Chinese college students attending a public university in China (Southeast). Out of an initial sample of 174 participants who completed surveys at Time 1, 152 (92 females \& 60 males) completed surveys at Time 2. All participants were enrolled in a psychology course and received extra credit for participation. Ages ranged from 18 to 22 years of age, with a mean age of $18.3(\mathrm{SD}=.8)$ years.

\section{Measures}

Personal growth initiative. Personal growth initiative was measured by the Personal 
Growth Initiative Scale-II (Robitschek et al., 2012). The PGIS-II is a 16-item measure made up of four subscales, namely, Readiness for Change (RC), Planfulness (P), Using Resources (UR), and Intentional Behavior (IB). Respondents are asked to rate their agreement to items across a 6point Likert-type scale. A Chinese adapted version of the PGIS-II (Yang \& Chang, 2014) was used. Reliabilities for the PGIS scales in the present sample ranged from .79 (RC at Time 1) to .86 ( $\mathrm{P}$ at Time 2). Higher scores on these subscales indicate greater personal initiative.

Life satisfaction. Life satisfaction was measured by the Satisfaction With Life Scale (SWLS; Diener, Emmons, Larsen, \& Griffin, 1985). The SWLS is a 5-item measure of life satisfaction. Respondents are asked their agreement to these items across a 7-point Likert-type scale. A Chinese adapted version of the SWLS (Chen \& Yang, 2003) was used. Reliabilities for the SWLS in the present sample were .92 (Time 1) and .90 (Time 2). Higher scores on this scale indicate greater life satisfaction.

\section{Procedure}

Approval for the study was obtained from the Institutional Review Board prior to data collection. All participants were measured for their personal growth initiative and life satisfaction at Time 1, as well as Time 2 (15 weeks apart). Participants for the present study received extra credit upon completion of the survey. All participants provided written, informed consent.

\section{Results}

Mean scores on the present set of measures at Time 1 and Time 2 are presented in Table 1. As the table shows, there were no significant differences on any of the measures across the two time points. Correlations between each of the present study measures are presented in Table 2. Overall, the correlations among the measures were generally significant and in the expected direction.

\section{Examining Personal Growth Initiative as a Predictor of Subsequent Life Satisfaction}

To examine if personal growth initiative at Time 1 predicts changes in life satisfaction at Time 2, we conducted a hierarchical regression analysis in which we initially controlled for demographic variables (viz., age \& sex) in Step 1, followed by life satisfaction at Time 1 in Step 2, the personal growth initiative set at Time 2 in Step 3, and the personal growth initiative set at Time 1 in Step 4. We calculated effect sizes to determine whether personal growth initiative accounted for small, medium, or large amounts of the variance in life satisfaction. 
Results of this analysis predicting life satisfaction at Time 2 are presented in Table 3. As the table shows, the set of demographic variables in Step 1 was not found to account for any significant amount of variance, $\mathrm{F}(2,149)=.42$, n.s. However, when life satisfaction at Time 1 was entered in Step 2, it was found to account for a large $\left(\mathrm{f}^{2}=.53\right) 35 \%$ of unique variance in life satisfaction at Time $2, \mathrm{~F}(1,148)=79.85, \mathrm{p}<.001$. Furthermore, the inclusion of personal growth initiative at Time 2 was found to account for a small-medium $\left(f^{2}=.10\right) 9 \%$ of additional unique variance in life satisfaction at Time $2, \mathrm{~F}(4,144)=6.10, \mathrm{p}<.001$. Within the predictor set, planfulness $(\beta=.24, \mathrm{p}<.05)$ was found to be the only significant unique predictor. Lastly, and importantly, the inclusion of personal growth initiative at Time 1 was found to account for a small-medium $\left(f^{2}=.09\right) 8 \%$ of additional unique variance in life satisfaction at Time $2, \mathrm{~F}(4,140)$ $=5.56, \mathrm{p}<.001$. Within the predictor set, planfulness $(\beta=.24, \mathrm{p}<.05)$ and (lack of) intentional behavior $(\beta=-.35, \mathrm{p}<.001)$ were found to be the only significant unique predictors. Overall, these results provide some support for the notion that personal growth initiative plays an important role in predicting future life satisfaction, but not always in the manner predicted by personal growth initiative theory.

\section{Examining Life Satisfaction as a Predictor of Personal Growth Initiative Processes}

To examine if life satisfaction at Time 1 predicts changes in each of the four personal growth initiative processes at Time 2 , we conducted a set of hierarchical regression analyses in which we initially controlled for demographic variables (viz., age \& sex) in Step 1, followed by the specific personal growth initiative dimension at Time 1 in Step 2, life satisfaction at Time 2 in Step 3, and life satisfaction at Time 1 in Step 4. Overall, the results of these analyses consistently failed to provide support for the notion that life satisfaction is important in predicting future personal growth initiative (see Table 4).

Discussion

An important goal of the present study was to examine the temporal relations between personal growth initiative and life satisfaction in college students. As mentioned earlier, personal growth initiative has been conceptualized as a positive psychological construct that is central to promoting mental health (Robitschek \& Keyes, 2009). Yet, a key limitation with past findings indicating a reliable cross-sectional association between personal growth initiative and life satisfaction is that it did not clarify if personal growth initiative is prospectively associated with life satisfaction.

This article is protected by copyright. All rights reserved 


\section{Some Evidence for Personal Growth Initiative as an Important Antecedent to Life Satisfaction, But Not Vice Versa}

Beyond the finding of a reliable association between personal growth initiative and life satisfaction at two different points in time, the present findings affirm the notion that personal growth initiative is an important prospective predictor of life satisfaction in college students. Specifically, we found that personal growth initiative (as a set) accounted for a significant amount of additional unique variance in life satisfaction 15 weeks later, even after controlling for baseline life satisfaction and concurrent personal growth initiative. In particular, two personal growth initiative dimensions emerged as significant predictors of life satisfaction, namely, intentional behavior and planfulness.

First, and foremost, we found that within the personal growth initiative set, intentional behavior at Time 1 was uniquely associated with later life satisfaction in students at Time 2 . Interestingly, however, the pattern obtained indicated that greater intentional behavior was prospectively associated with lower life satisfaction in students. This raises the possibility that although intentional behavior, the conscious and constant pursuit of personal growth, is believed to reflect a positive process (Robitschek et al., 2012), it may lead to more distressing, rather than satisfying experiences over time. One possible explanation for this is that individuals may pursue too many paths to achieve personal growth. Interestingly, findings from past studies have shown that positively motivated individuals (e.g., optimists), compared to negatively motivated individuals (e.g., pessimists), are more likely to become distressed when engaged in the challenges of pursuing multiple goals (Cohen et al., 1999; Segerstrom, 2001). This appears to be due to the fact that many of the goals we pursue engender competing pathways to achieve success or personal growth (Emmons \& King, 1988).

In addition, one might be able to argue that the emergence of conflicting goals over time is likely to be further intensified by a need to consider personal growth goals in the context of other individuals. For example, individuals might experience challenge, if not threat, when they find themselves having to modify or change their personal growth goals to accommodate the goals of others important to them in order to maintain a positive relationship (Tomaka, Blascovich, Kelsey, \& Leitten, 1993). Consistent with this notion, Chen, Cheung, Bond, and Leung (2006) found that greater appreciation of social complexity, the notion that one's behavior must often change with the social context, was associated with lower levels of life satisfaction 
among students. This pattern might be especially true for individuals coming from collectivistic societies (e.g., China, Japan, \& Korea; Markus \& Kitayama, 1991; Oishi, Diener, Lucas, \& Suh, 1999), where individuals must harmoniously evaluate their intentional behaviors and goals in the context of the intentions and goals of significant others in their lives (e.g., parents, family; Chang \& Yang, 2016; Wu, 2013). Thus, one important practical implication of this finding is that the function of personal growth initiative dimensions such as intentional behavior might need to be situated as a complex function of both time and culture. Thus, it would be useful to determine in future studies if efforts to alleviate strain associated with intentional behavior among Chinese students might help them develop a stronger sense of life satisfaction over time.

Furthermore, given that the association between intentional behavior at Time 1 only emerged as a significant predictor of life satisfaction at Time 2 after controlling for other variables, this pattern raises the possibility that the (negative) role of intentional behavior on future life satisfaction might be suppressed by a combination of concurrent and subsequent personal growth dimensions as well as concurrent life satisfaction. Accordingly, for example, it would be important in future studies to control for overlap among the personal growth dimensions when testing prediction models to determine if unique, and sometimes unexpected, effects emerge in accounting for psychological adjustment.

Alternatively, consistent with personal growth initiative theory, we found planfulness at Time 1 was uniquely found to predict greater life satisfaction in students at Time 2 . That is, students who engaged in greater efforts to make realistic changes for personal growth were found to be more satisfied with their life 15 weeks later. It may be that the ability to identify realistic pathways to promote growth provides a useful strategy by which students can better match their skills and abilities to a specific growth goal in order to ensure greater likelihood of success (Azizli, Atkinson, Baughman, \& Giammarco, 2015). Interestingly, Yang and Chang (2016) found that even after controlling for hope, planfulness continued to be an important unique correlate of life satisfaction in college students. That said, it would be important to determine in future studies if planfulness remains an important prospective predictor of life satisfaction after controlling for other important goal-directed constructs like hope.

In contrast to the finding that personal growth initiative was an important prospective predictor of later life satisfaction, we failed to find evidence to support the possibility that life satisfaction was an important prospective predictor of later personal growth initiative. This 
pattern is consistent with findings obtained from a recent study indicating that markers of subjective well-being (viz., positive affect) are likely to be better prospective predictors associated with "broadening" our attention and thought action-repertoires (e.g., greater number of things imagined doing when feeling good or being satisfied with life), than they are likely to be prospective predictors associated with "building" personal coping skills and character strengths (cf., Frederickson, 2001). Noteworthy, similar to the conceptualization of personal growth initiative as a skills-based process, Chang (2017) found that skills-based social problemsolving dimensions (e.g., rational problem solving, avoidance style) were not significantly associated with baseline positive affect measured two months earlier. In contrast, in support of broadening effects, Chang found that baseline affect was prospectively associated with greater positive problem orientation and lower negative problem orientation two months later. Finally, it is worth nothing the importance of considering our use of a 15 -week period. Specifically, it would be important in future studies to determine if the use of a shorter or longer time fame might result in different patterns.

\section{Concluding Comment}

Findings from the present study are the first to provide support for the notion that personal growth initiative is an important prospective predictor of positive psychological adjustment in adults. Specifically, intentional behavior has a negative impact on life satisfaction, while planfulness has a positive one. In contrast, we were able to show that life satisfaction was not an important prospective predictor of personal growth initiative in adults. Future research might focus on identifying important antecedents to personal growth initiative that might be leveraged to cultivate this important positive psychological process in adults.

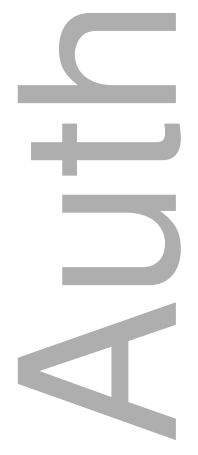




\section{References}

Azizli, N., Atkinson, B. E., Baughman, H. M., \& Giammarco, E. A. (2015). Relationships between general self-efficacy, planning for the future, and life satisfaction. Personality and Individual Differences, 82, 58-60.

Chang, E. C. (2017). Applying the broaden-and-build model of positive emotions to social problem solving: Does feeling good (vs. feeling bad) influence problem orientation, problem-solving skills, or both? Journal of Social and Clinical Psychology, 36, 380-395.

Chang, E. C., Downey, C. A., Hirsch, J. K., \& Lin, N. J. (Eds.). (2016). Positive psychology in racial and ethnic minority groups: Theory, research, and practice. Washington, DC: American Psychological Association.

Chang, E. C., \& Yang, H. (2016). Personal and family growth initiative as predictors of study engagement in Chinese and American college students: Is there any evidence for group differences? Personality and Individual Differences, 102, 186-189.

Chen, J., \& Yang, H. (2003). Hangzhoushi dxuesheng shenghuomanyigan diaocha [A survey on college students' satisfaction with life]. Chinese Journal of Clinical Psychology, 11, 202203.

Chen, S. X., Cheung, F. M., Bond, M. H., \& Leung, J.-P. (2006). Going beyond self-esteem to prediet life satisfaction: The Chinese case. Asian Journal of Social Psychology, 9, 24-35.

Cohen, F., Kearney, K. A., Zegans, L. S., Kemey, M. E., Neuhaus, J. M., \& Stites, D. P. (1999). Differential immune system changes with acute and persistent stress for optimists vs. pessimists. Brain, Behavior, and Immunity, 13, 155-174.

Diener, E. (1984). Subjective well-being. Psychological Bulletin, 95, 542-575.

Diener, E., Emmons, R. A., Larsen, R. J., \& Griffin, S. (1985). The Satisfaction With Life Scale. Journal of Personality Assessment, 49, 71-75.

Diener, E.,Oishi, S., \& Lucas, R.. E. (2003). Personality, culture, and subjective well-being: Emotional and cognitive evaluations of life. Annual Review of Psychology, 54, 403-425.

Emmons, R. A., \& King, L. A. (1988). Conflict among personal strivings: Immediate and longterm implications for psychological and physical well-being. Journal of Personality and Social Psychology, 54, 1040-1048.

Fredrickson, B. L. (2001). The role of positive emotions in positive psychology: The broadenand-build theory of positive emotions. American Psychologist, 56, 218-226. 
Luhmann, M., \& Hennecke, M. (2017). The motivational consequences of life satisfaction. Motivation Science, 3, 51-75.

Markus, H. R., \& Kitayama, S. (1991). Culture and the self: Implications for cognition, emotion, and motivation. Psychological Review, 98, 224-253.

Oishi, S., Diener, E. F., Lucas, R. E., \& Suh, E. M. (1999). Cross-cultural variations in predictors of life satisfaction: Perspectives from needs and values. Personality and Social Psychology Bulletin, 25, 980-990.

Robitschek, C. (1998). Personal growth initiative: The construct and its measure. Measurement and Evaluation in Counseling and Development, 30, 183-198.

Robitschek, C., Ashton, M. W., Spering, C. C., Geiger, N., Byers, D., Schotts, G. C., \& Thoen, M. (2012). Development and psychometric properties of the Personal Growth Initiative Scale - II. Journal of Counseling Psychology, 59, 274-287.

Robitschek, C., \& Keyes, C. L. M. (2009). Keyes' model of mental health with personal growth initiative as a parsimonious predictor. Journal of Counseling Psychology, 56, 321-329.

Ryff, C. D., \& Keyes, C. L. M. (1995). The structure of psychological well-being revisited. Journal of Personality and Social Psychology, 69, 719-727.

Segerstrom, S. C. (2001). Optimism, goal conflict, and stressor-related immune change. Journal of Behavioral Medicine, 24, 441-467.

Seligman, M. E. P., \& Csikszentmihalyi, M. (2000). Positive psychology: An introduction. American Psychologist, 55, 5-14.

Shorey, H. S., Little, T. D., Snyder, C. R., Kluck, B., \& Robitschek, C. (2007). Hope and personal growth initiative: A comparison of positive, future-oriented constructs. Personality and Individual Differences, 43, 1917-1926.

Snyder, C. R., Harris, C., Anderson, J. R., Holleran, S. A., Irving, L. M., Sigmon, S. T., ... \& Harney, P. (1991). The wills and the ways: Development and validation of an individual differences measure of hope. Journal of Personality and Social Psychology, 60, 570-585.

Stevic, C. R., \& Ward, R. M. (2008). Initiating personal growth: The role of recognition and life satisfaction on the development of college students. Social Indicators Research, 89, 523534.

Tomaka, J., Blascovich, J., Kelsey, R. M., \& Leitten, C. L. (1993). Subjective, physiological, and behavioral effects of threat and challenge appraisal. Journal of Personality and Social 
Psychology, 65, 248-260.

Wu, M.-Y. (2013). The concept of guan in the Chinese parent-child relationship. In C.-C. Yi

(Ed.), The psychological well-being of East Asian youth (pp. 29-49). New York: Springer Science + Business Media.

Yang, H., \& Chang, E. C. (2014). Examining the structure, reliability, and validity of the Chinese Personal Growth Initiative Scale-II: Evidence for the importance of intentional selfchange among Chinese. Journal of Personality Assessment, 96, 559-566.

Yang, H., \& Chang, E. C. (2016). Is the PGIS-II redundant with the Hope Scale?: Evidence for the utility of the PGIS-II in predicting psychological adjustment in adults. Personality and Individual Differences, 94, 124-129.

Table 1

Mean Differences in Personal Growth Initiative and Life Satisfaction in Students Across Time 1 and Time 2 (15 Weeks Later)

\begin{tabular}{lccccc}
\hline Measure & \multicolumn{2}{c}{ Time 1 } & Time 2 & \\
& $\mathrm{M}$ & $\mathrm{SD}$ & $\mathrm{M}$ & $\mathrm{SD}$ & $\mathrm{t}(151)$ \\
\hline Personal Growth Initiative & & & & & \\
$\quad$ Readiness for Change & 4.24 & .87 & 4.30 & .79 & -.89 \\
Planfulness & 4.37 & .77 & 4.36 & .81 & .27 \\
Using Resources & 4.59 & .86 & 4.53 & .86 & .72 \\
Intentional Behavior & 4.73 & .69 & 4.64 & .74 & 1.40 \\
Life Satisfaction & 4.75 & 1.07 & 4.69 & 1.22 & .67 \\
\end{tabular}

Note. $\mathrm{N}=152$. 


\section{Table 2}

Correlations Between All Study Measures in Students

\begin{tabular}{|c|c|c|c|c|c|c|c|c|c|c|}
\hline Measures & 1 & 2 & 3 & 4 & 5 & 6 & 7 & 8 & 9 & 10 \\
\hline
\end{tabular}

1. PGIS-II-RC-T1 --

2. PGIS-HI-P-T1 .67*** --

3. PGIS-II-UR-T1 .39*** .44*** --

4. PGIS-II-IB-T1 .60*** .69*** .53*** --

$\begin{array}{llllll} & \text { 5. SWLS-T1 } & .40 * * * & .37 * * * & .16 * & .41 * * *\end{array}$

6. PGIS-II-RC-T2 $\quad .40 * * * \quad .48 * * * \quad .17 * \quad .30 * * * \quad .31 * * * \quad--$

$\begin{array}{llllllll}\text { 7. PGIS-II-P-T2 } & .40 * * * & .59 * * * & .20 * & .32 * * * & .32 * * * & .78 * * * & --\end{array}$

8. PGIS-II-UR-T2 $\quad .21 * \quad .26 * * * \quad .31 * * * \quad .21 * \quad .11^{*} \quad . \quad .53 * * * \quad .56 * * * \quad$--

9. PGIS-II-IB-T2 $\quad .37 * * * \quad .46 * * * \quad .22 * * \quad 145 * * * \quad .38 * * * \quad .72 * * * \quad .74 * * * \quad .65 * * * \quad--$

$\begin{array}{lllllllllll}10 . \mathrm{SWLS}-\mathrm{T} 2 & .20 * & .31 * * * & -.01 & .13 & .59 * * * & .42 * * * & .47 * * & .26 * * * & .47 * * * & --\end{array}$

Note. $\mathrm{N}=152$. PGIS-II = Personal Growth Initiative Scale-II; RC = Readiness for Change; $\mathrm{P}=$ Planfulness; UR = Using Resources; IB = Intentional Behavior; SWLS = Satisfaction With Life Scale; T1 = Time 1; T2 = Time 2 (15 weeks later).

$* \mathrm{p}<.05 . * * * \mathrm{p}<.001$.

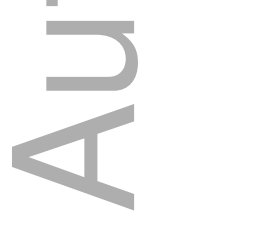

This article is protected by copyright. All rights reserved 
Table 3

Hierarchical Regression Analyses Showing Amount of Variance in Life Satisfaction at Time 2 Accounted for by Personal Growth Initiative at Time 1, After Controlling for Life Satisfaction at Time 1 and Personal Growth Initiative at Time 2

\begin{tabular}{|c|c|c|c|c|}
\hline Outcome/Predictor & $\beta$ & $\mathrm{R}^{2}$ & $\Delta \mathrm{R}^{2}$ & $\mathrm{~F}$ \\
\hline \multicolumn{5}{|l|}{ Time 2 Life Satisfaction } \\
\hline \multicolumn{2}{|l|}{ Step 1: Demographics } & .01 & -- & .42 \\
\hline Age & .04 & & & \\
\hline Sex & .06 & & & \\
\hline Step 2: Time 1 Life Satisfaction & $.60 * * *$ & .35 & .35 & $79.85 * * *$ \\
\hline \multicolumn{2}{|c|}{ Step 3: Time 2 Personal Growth Initiative } & .45 & .09 & $6.10 * * *$ \\
\hline Readiness for Change & .01 & & & \\
\hline Planfulness & $.24 *$ & & & \\
\hline Using Resources & -.06 & & & \\
\hline Intentional Behavior & .15 & & & \\
\hline \multicolumn{2}{|c|}{ Step 4: Time 1 Personal Growth Initiative } & .52 & .08 & $5.56 * * *$ \\
\hline Readiness for Change & -.11 & & & \\
\hline Planfulness & $.24 *$ & & & \\
\hline Using Resources & -.06 & & & \\
\hline Intentional Behavior & $-.35 * * *$ & & & \\
\hline
\end{tabular}

Notes. $\mathrm{N}=152$.

$* \mathrm{p}<.05 . * * * \mathrm{p} \leq .001$. 
Table 4

Hierarchical Regression Analyses Showing Amount of Variance in Each Personal Growth Initiative Dimension at Time 2 Accounted for by Life Satisfaction at Time 1, After Controlling for Baseline Personal Growth Initiative Dimension at Time 1 and Life Satisfaction at Time 2

\begin{tabular}{lllll}
\hline Outcome/Predictor & $\beta$ & $\mathrm{R}^{2}$ & $\Delta \mathrm{R}^{2}$ & $\mathrm{~F}$ \\
\hline
\end{tabular}

Time 2 Readiness for Change

Step 1: Demographics

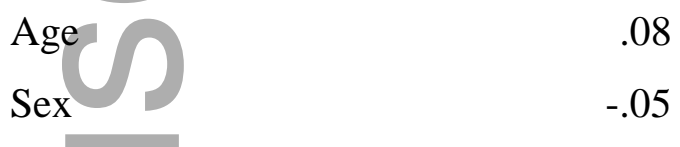

Step 2: Time 1 Readiness for Change

Step 3: Time 2 Life Satisfaction

Step 4: Time 1 Life Satisfaction

Time 2 Planfulness

Step 1: Demographics

Age

Sex

Step 2: Time 1 Planfulness

Step 3:Time 2 Life Satisfaction

Step 4: Time 1 Life Satisfaction

Time 2 Using Resources

Step 1: Demographics

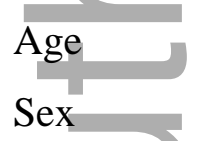

Step 2: Time 1 Using Resources

Step 3: Time 2 Life Satisfaction

Step 4: Time 1 Life Satisfaction

Time 2 Intentional Behavior

Step 1: Demographics

Age

.08

$-.05$

$-.03$

.11

.02

$-.07$

.13

$-.02$

$-.03$
.01

.60

$.42 * * * \quad .18$

.30

.30

.01

$--$

.96

$.59 * * *$

.35

.34

$78.36^{* * *}$

$.32 * * *$

.45

.09

$24.85^{* * *}$

.45

.00

.68

.02

--

1.19

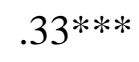

.12

.11

$17.78 * * *$

$.26 * * *$

.19

.07

$12.22 * * *$

.19

.00

.13

.02

--

1.17 
Sex

.06

Step 2: Time 1 Intentional Behavior

$.50 * * *$

.24

.23

$43.95^{* * *}$

Step 3: Time 2 Life Satisfaction

$.41 * * *$

.41

.16

$40.61 * * *$

Step 4: Time 1 Life Satisfaction

$-.07$

.41

.00

.58

Notes. $\mathrm{N}=152$.

$* * * \mathrm{p} \leq .001$
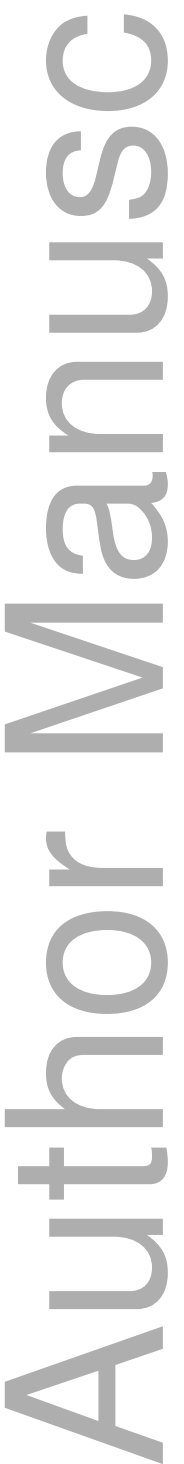

This article is protected by copyright. All rights reserved 